\title{
ENHANCEMENT OF TURBULENT AIRFLOW AND HEAT TRANSFER THROUGH A RECTANGULAR MICROCHANNEL WITH DIFFERENT TYPES OF BAFFLES
}

\author{
Sandip Saha ${ }^{1}$, Santanu Raut ${ }^{2}$, Apurba Narayan Das ${ }^{3}$ \\ ${ }^{I}$ Department of Mathematics, National Institute of Technology Silchar, Silchar-788010, Assam, India \\ 2 Department of Mathematics, Mathabhanga College, Cooch Behar-736146, West Bengal, India \\ ${ }^{3}$ Department of Mathematics, Alipurduar University, Alipurduar-736121, West Bengal, India \\ sandip.tfgss@gmail.com,rautsantanu99@gmail.com,apuraiganj1964@gmail.com
}

Received: 11 March 2021; Accepted: 3 August 2021

\begin{abstract}
The objective of this work is to discuss the turbulent air hydro-thermal phenomena over a rectangular microchannel with different types of baffle (rectangular, triangular, and trapezoidal) mounted on both the walls of the microchannel. The finite volume method with the second order upwind scheme has been utilized to discretize the governing equations and to study the turbulent airflow characteristics; the SST k- $\omega$ turbulence model has been adopted. For nine different cases, the different characteristics of fluid flow phenomena and thermal behaviour with the variations in the Reynolds number ranging from [5,000-25,000] and for three different values of inter baffle spacing have been studied in this manuscript. Due to the presence of baffle, it is revealed that the vortex arises on the upper wall and the thermal phenomena enhances with the decrease in inter baffle spacing.
\end{abstract}

MSC 2010: 74A15, 80A05

Keywords: turbulent air flow, heat transfer, local Nusselt number, friction coefficient

\section{Nomenclature}

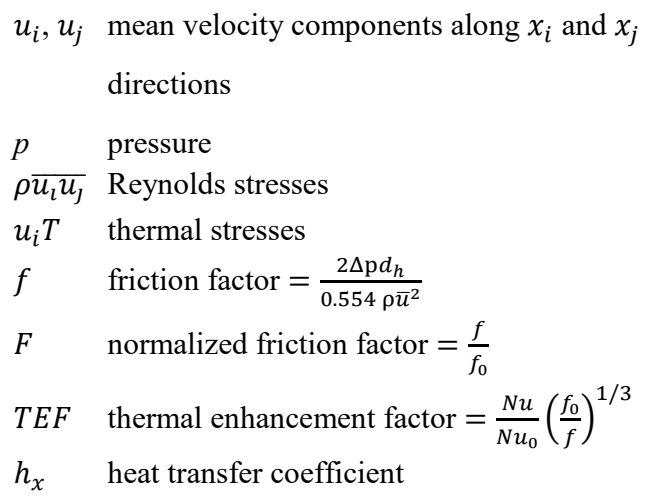

$\begin{array}{ll}R e & \text { Reynolds number }=\frac{\rho \bar{u} d_{h}}{\mu} \\ N u_{x} & \text { local Nusselt number }=\frac{h_{x} d_{h}}{k_{f}} \\ \Delta p & \text { absolute pressure drop }=\left|\left(p_{2}-p_{1}\right)\right| \\ d_{h} & \text { hydraulic diameter } \\ \bar{u} & \text { average velocity } \\ x, y & \text { Cartesian coordinate } \\ u_{\text {in }} & \text { reference velocity } \\ C_{f} & \text { local skin friction coefficient }=\frac{\tau_{w}}{2 \rho \bar{u}^{2}} \\ C_{p} & \text { pressure coefficient }\end{array}$




$\begin{array}{llll}k_{f} & \text { thermal conductivity }\left[\mathrm{Wm}^{-1} \mathrm{~K}^{-1}\right] & \tau_{w} & \text { wall shear stress } \\ p_{1} & \text { pressure at inlet section } & T & \text { temperature } \\ k_{t u r} & \text { kinetic turbulent energy } & S S T & \text { shear stress transport } \\ p_{2} \quad \text { pressure at outlet section } & \varphi_{k}, \varphi_{\omega} \text { effective diffusivity of } k \text { and } \omega \\ N u \quad \text { average Nusselt number }=\frac{1}{0.554} \int_{0}^{L} N u_{x} d x & Q_{\omega} & \text { user defined source term } \\ N u_{\text {avg }} & \text { normalized average Nusselt number }=\frac{N u}{N u_{0}} & C_{p} & \text { average pressure coefficients } \\ L D A & \text { Laser Doppler Anemometry } & D_{\omega} & \text { cross diffusion terms } \\ R_{k}, R_{\omega} & \text { turbulence dissipation term of } k, \omega & \omega & \text { dissipation rate } \\ \rho \quad \text { density }\left[\mathrm{kg} \cdot \mathrm{m}^{-3}\right] & \alpha^{*} & \text { damps of turbulent viscosity } \\ \mu \quad \text { dynamic viscosity } & & \end{array}$

\section{Introduction}

In last few decades, heat exchange has become one of the most important topics in the engineering and scientific fields due to its wide applications in commercial products such as air conditioners, room coolers, chemical reactors, etc [1-3]. Generally, heat passes through various modes like convection, conduction and radiation. The most effective and popularly used technique is to place baffles in the perpendicular direction of the flow in a channel. In general, a very complex flow pattern has been found in the complex geometry formed for placing baffles in the system [4-7].

In 2021, with Y-shaped triangular baffles, Pandey and Singh [4] studied the turbulent flow phenomena of heat transmission in a tube for different values of $R e$. At $R e=3,000$, they concluded that the value of TEF become 3.25 times than that of the case of smooth channel. Nagarajan and Sivashanmugam [5] observed the thermal characteristics of turbulent flow through a circular tube with a helical baffle. They stated that rate of thermal exchange increases with the increase in the value of $R e$. In the presence of an airfoil baffle, Gururatana and Skullong [6] studied the turbulent flow for various baffle angles. They stated that the value of TEF becomes maximum when the baffle angle is $45^{\circ}$. In a rectangular cavity, Tang and Zhu [7] investigated the hydro-thermal phenomena of turbulent flow with crossed baffles. They revealed that the rate of thermal exchange increases by 10 to $13.5 \%$ compared to the case of the rectangular baffled channel. Over a rectangular duct, Sripattanapipat and Promvonge [8] studied the thermal enhancement in the presence of diamond and rectangular shaped baffles. They found that the presence of diamond shape baffle provides better heat exchange than the case of a rectangular baffle. Over a reactangular microchannel, Habib et al. [9] experimentally studied the turbulent hydro-thermal phenomena with segmented baffles and revealed that the increase in the baffle height causes the increase in $\Delta p$.

Siddiqui and Kamran [10] experimentally and numerically studied the heat transfer enhancement through a rectangular microchannel with flat and inclined baffles. They stated that the value of $N u$ increases when the baffle is perpendicular to the walls. In 2004, Demartini et al. [11] experimentally and numerically studied the fluid 
flow phenomena through a rectangular microchannel with two plane baffles. At $x=0.525 \mathrm{~m}$ and $R e=87,300$, they found that the velocity becomes $4.36 \mathrm{~m} / \mathrm{s}$ times of the input velocity. In 2020, Saha et al. [12] performed a numerical study on hydrothermal phenomena through a rectangular channel with two, three and four plane and trapezoidal shaped baffles. At $R e=87,300$, they stated that in the case of rectangular baffles, the axial velocity becomes 2.949 times that of the input velocity, while in case of trapezoidal baffles, it becomes 6 times of input velocity. In 2021, based on the geometry of Demartini et al. [11], considering sixteen alternate formations of the baffle (plane, trapezoidal, triangular and isosceles), Saha et al. [13] numerically studied turbulent air hydrothermal phenomena. At a fixed Re, they demonstrated that the value of $\mathrm{Nu}$ becomes maximum in case of trapezoidal-triangular baffles. Similar types of work are available in the literature [14-23].

The present work is an extension of the experimental work of Demartini et al. [11] and the numerical works of Saha et al. [12,13]. In the aforesaid work [11], the phenomena of heat transfer has not been considered and Saha et al. [12,13] studied the hydrothermal phenomena keeping the location of the baffles fixed. From the above literature survey, it is clear that no work has been done to describe the different kinds of hydrothermal phenomena so far, considering the variations in inter baffle spacing, which provides us with enough motivation to carry forward the present study.

\section{Purpose of the study}

The purpose of this study has been prescribed as follows:

i) to study the effect of nine different forms of baffle (plane, triangular and trapezoidal) on different hydro-thermal characteristics of turbulent air flow phenomena,

ii) to observe the effect of inter baffle spacing (11, 12 and 13) on the heat transfer characteristics and flow phenomena,

iii) to discuss the profiles of $N u_{\text {avg }}$ and $T E F$ for different values of $R e$.

\section{Flow geometry and dimensions}

The study of the problems of the flow phenomena through a rectangular microchannel has become more interesting in heat transfer engineering as well as in fluid mechanics [10-16]. A rectangular micro-channel with nine different kinds of baffle configurations, such as, plane baffles at a distance $l 1$ [case-1(a)], $l 2$ [case-1(b)] and $l 3$ [case-1(c)], triangular baffles at a distance $l 1$ [case-2(a)], $l 2$ [case-2(b)] and $l 3$ [case-2(c)], trapezoidal baffles at a distance $l 1$ [case-3(a)], $l 2$ [case-3(b)] and $l 3$ [case-3(c)], where the baffles are alternately mounted on lower and upper channel walls, have been presented in the Figures 1a-i. Each of the channels consists of four sections, namely inlet, outlet, lower and upper walls. The radiation of heat transfer, 
body forces, viscous dissipation and Buoyancy effects are neglected in this work. Based on the experimental work of Demartini et al. [11] the dimensions of the geometry and the fluid properties have been considered. To model the geometry (Fig. 1) and for the purpose of simulation, FLUENT software has been employed.

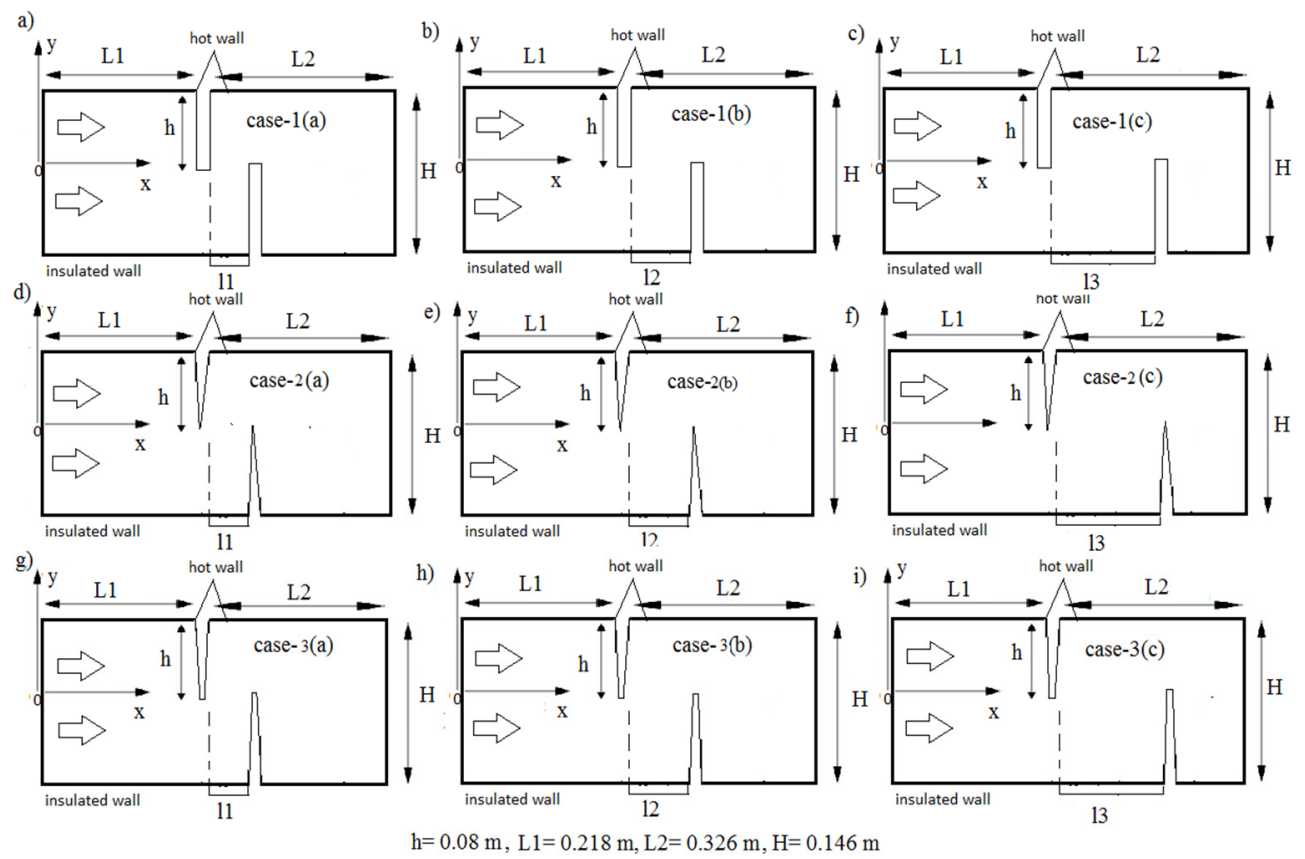

Fig. 1. Rectangular micro-channels in presence of plane [1(a-c)], triangular [2(a-c)] and trapezoidal $[3(\mathrm{a}-\mathrm{c})]$ baffles

The two-dimensional flow is assumed to be incompressible and Newtonian. In this work, the analysis of fluid flow phenomena and heat transfer, for the variations in the type of baffle and for different locations of the second baffle keeping the first baffle fixed, has been done.

\section{Mathematical equations}

Hydro-thermal characteristics of turbulent air flow are governed by the following continuity (equation (1)), momentum (equation (2)) and energy (equation (3)) equations [10-23]:

$$
\begin{gathered}
\frac{\partial\left(\rho \cdot u_{i}\right)}{\partial x_{i}}=0 \\
\frac{\partial\left(\rho \cdot u_{i} \cdot u_{j}\right)}{\partial x_{i}}=-\frac{\partial p}{\partial x_{i}}+\frac{\partial}{\partial x_{j}}\left[\mu\left(\frac{\partial u_{i}}{\partial x_{j}}+\frac{\partial u_{j}}{\partial x_{i}}-\frac{2}{3} \delta_{i j} \frac{\partial u_{i}}{\partial x_{j}}\right)\right] \frac{\partial}{\partial x_{j}}\left(-\rho \cdot \overline{u_{\imath} u_{J}}\right)
\end{gathered}
$$




$$
\frac{\partial\left(\rho \cdot u_{i} \cdot T\right)}{\partial x_{j}}=\frac{\partial}{\partial x_{j}}\left[\left(\frac{\mu}{P r}-\frac{\mu_{t}}{P r_{t}}\right) \frac{\partial T}{\partial x_{j}}\right]
$$

For analyzing the hydro-thermal phenomena, near the walls and far away from the walls, the $S S T k-\omega$ model is suitable due to the presence of $D_{\omega}$ term, blending function. To predict the accurate results, this model is used to transform the inner region of boundary layer to the $k-\omega$ model at a higher $R e$. The SST $k-\omega$ model $[12-14,16,17,22]$ contains two transport equations (4) and (5) involving $k_{t u r}$ and $\omega$ and those are:

$$
\begin{aligned}
& \frac{\partial\left(\rho \cdot k_{t u r} \cdot u_{i}\right)}{\partial x_{i}}=\frac{\partial}{\partial x_{j}}\left[\varphi_{k}\left(\frac{\partial k_{t u r}}{\partial x_{j}}\right)\right]-\rho \overline{u_{\imath}^{\prime} u_{\jmath}^{\prime}} \frac{\delta u_{j}}{\delta x_{i}}+R_{k}-\rho * 0.072 \\
& *\left(1+0.05 * F\left(M_{t}\right)\right) f_{\beta}{ }^{*} k \omega \\
& \frac{\partial\left(\rho . \omega . u_{i}\right)}{\partial x_{i}}=\frac{\partial}{\partial x_{j}}\left[\varphi_{\omega}\left(\frac{\partial \omega_{t u r}}{\partial x_{j}}\right)\right]+\frac{\alpha_{\infty}}{\alpha^{*}}\left(\frac{\left(0.024+\frac{\rho . k}{2.95 \mu . \omega}\right)}{\left(1+\frac{\rho . k}{2.95 \mu . \omega}\right)}\right)+R_{\omega}-\rho \\
& \text { * } 0.072 \\
& *\left(1-1.3125 *\left(\frac{\left(\frac{4}{15}+\left(\frac{\rho . k}{8 \mu \cdot \omega}\right)^{4}\right)}{\left(1+\left(\frac{\rho . k}{8 \mu \cdot \omega}\right)^{4}\right)}\right) * F\left(M_{t}\right)\right. \\
& \left.*\left(\frac{1+70 *\left(\left|\frac{\left(\Omega_{i j} \Omega_{j k} S_{k i}\right.}{\left(\beta_{\infty}^{*} \cdot \omega\right)^{3}}\right|\right)}{1+80 *\left(\left|\frac{\left(\Omega_{i j} \Omega_{j k} S_{k i}\right.}{\left(\beta^{*}{ }_{\infty} \cdot \omega\right)^{3}}\right|\right)}\right)\right) \omega^{2}+Q_{\omega}
\end{aligned}
$$

with $\varphi_{k}=\mu+\alpha^{*} \frac{\rho . k}{\sigma_{k}}, \quad \varphi_{\omega}=\mu+\alpha^{*} \frac{\rho . \omega}{\sigma_{\omega}}$

For small values of $R e$, the coefficient $\alpha^{*}$ is written as:

$$
\alpha^{*}=\alpha_{\infty}^{*} \frac{\left(0.024+\frac{\rho . k}{6 \mu \cdot \omega}\right)}{\left(1+\frac{\rho . k}{6 \mu \cdot \omega}\right)}
$$

and for large values of $\operatorname{Re}, \alpha^{*}=\alpha_{\infty}^{*}=1$, and

$$
f_{\beta}{ }^{*}=\left\{\begin{array}{cc}
1, & A_{k}<0 \\
\frac{\left(1+680 * A_{k}{ }^{2}\right)}{\left(1+400 *{A_{k}}^{2}\right)}, & A_{k}>0
\end{array}\right.
$$




$$
F\left(M_{t}\right)= \begin{cases}0, & M_{t} \leq M_{t_{0}} \\ \frac{2 k}{\gamma R t}-(0.25)^{2}, & M_{t}>M_{t_{0}}\end{cases}
$$

For $R e \geq 10_{4}, N u_{0}$ taken as per Dittus and Boelter [17]:

$$
N u_{0}=0.023 * \operatorname{Re}^{0.8} * \operatorname{Pr}^{0.4}
$$

For $3 \times 10^{3} \leq R e \leq 5 \times 10^{6}, f_{0}$ has been taken as per Petukhov [18]:

$$
f_{0}=(0.79 * \ln R e-1.64)^{-2}
$$

\subsection{Boundary conditions and Numerical procedures}

According to the experimental result of Dermitini et al. [11] and the numerical work of Saha et al. [12], the hydrodynamic boundary conditions have been specified and the thermal boundary conditions have been chosen from the works of Siddiqui and Kamran [10], Saha et al. [12]. A uniform velocity distribution $\left(y=0 ; u_{i n}\right)$ has been prescribed at the inlet section and the fluid temperature has been set as $27^{\circ} \mathrm{C}$. No slip and no penetratation boundary conditions $(u=0 ; v=0)$ have been applied at the channel walls $(y=0 ; y=0.146 \mathrm{~m})$, and pressure outlet with zero gauge pressure has been taken as outlet boundary conditions. The upper and lower walls of the computational domain have been kept at $102^{\circ} \mathrm{C}$. To study the fluid flow and heat transfer phenomena in the physical domain, the commercial software FLUENT has been employed, and finite volume method has been used to solve the governing equations. The SIMPLE algorithm has been used to develop the primitive variables (pressure and velocity) of the governing equations and for convective terms, the second order upwind scheme has been adopted. To achieve the high velocity gradient, pressure and temperature, the grid has been chosen as non uniform which is highly dense near the baffles. In addition, for all the dependent variables, the convergence criteria have been set as $10^{-9}$.

\subsection{Test of grid and validation of code}

To assess the impact of grid sizes on the findings, a grid test has been conducted at $R e=87,300$ on a rectangular micro-channel like Demartini et al. [11], Saha et al. [12]. From Figure 2a, it is clearly seen that after 20,000 elements, the trade of $C_{p}$ becomes linear and constant, which indicates that 20,000 numbers of elements [11] are sufficient to carry out the future analysis. The validation of the code has been done on the same geometrical configuration and boundary conditions as set in the experimental work of Demartini et al. [11], where they mounted two plane baffles alternately on the lower and upper walls of the channel. At different locations and $R e=87,300$, Figures $2 \mathrm{~b}-\mathrm{e}$, show that the results of this work are too close to those 
of Demartini et al. [11], which provide enough confidence to continue the present study.
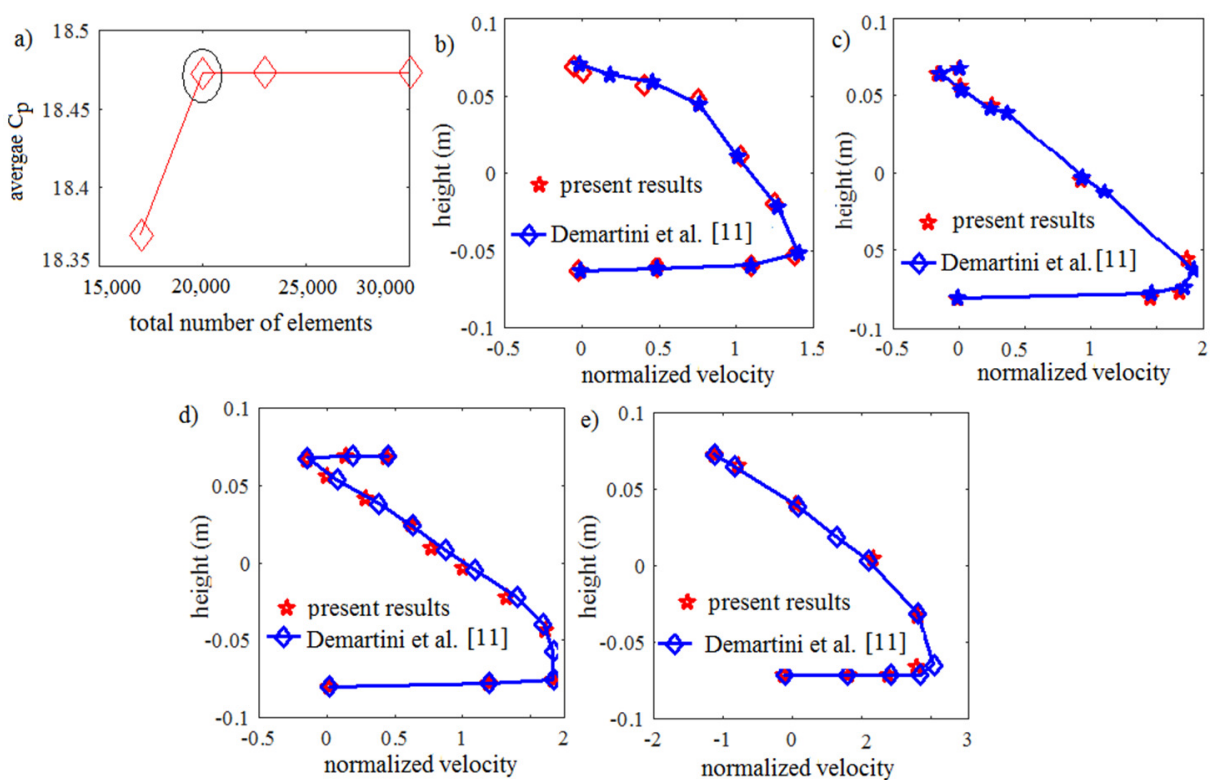

Fig. 2. Average $C_{p}$ vs total number of elements (a), variations of velocity profile at $x=0.159 \mathrm{~m}$ (b), $x=0.189 \mathrm{~m}(\mathrm{c}), x=0.289 \mathrm{~m}(\mathrm{~d})$ and $x=0.525 \mathrm{~m}$ (e)

\section{Results and discussions}

The focus of this section is to investigate the effect of different kinds of baffle and of the inter baffle spacing on the thermal behavior at $R e$ ranging between 5,000 and 25,000, in terms of velocity profile, $N u_{x}, N u_{\text {avg }}, F$ and $T E F$.

\subsection{Flow phenomena}

For all the considered cases of baffles and for three different positions of the baffles, viz., $l 1=0.071 \mathrm{~m}, l 2=0.142 \mathrm{~m}$ and $l 3=0.213 \mathrm{~m}$, Figures $3 \mathrm{a}-\mathrm{i}$ present the velocity profiles at $R e=5,000$. The results show the existence of three flow zones. The first zone is formed when the fluid flows with the reference velocity. When the flow comes in contact with the first baffle, the velocity profiles become more influenced and a second zone is formed at the upper face of the first baffle, where the rate of flow enhances due to the decrease of flow area. Lastly, at the downstream of the second baffle, the third zone is formed due to the flow of the air over the baffles towards the upper wall. As a result, the zone of recirculation exists (negative velocity values arise) at the third flow zone, which increases as $R e$ increases. The vortex shedding generated, due to the presence of baffles, affects the flow field 
and the vortex length. It is illustrated that the increase in inter baffle spacing [case-1(c), case-2(c), case-3(c)] causes the existence of a small recirculation zone, whereas the large recirculation zone exists at small values of inter baffle spacing [case-1(a), case-2(b), case-3(c)]. Figure 3 presents the numerical results of axial velocity profiles for three different values of inter baffle spacing and three different types of baffle at $x=0.189 \mathrm{~m}$ (Fig. 3a-c) i.e., ahead of first baffle. It is observed that as the flow approaches the first baffle, the direction of the flow velocity changes at the upper face of the first baffle. At location $x=0.289 \mathrm{~m}$, taken in between two baffles, Figures $3 d$-e present the velocity profiles for all the cases. In addition, at the downstream of the first baffle (at $x=0.289 \mathrm{~m}$ ), flow velocity becomes higher than that at the location $x=0.189 \mathrm{~m}$ and the negative velocity values are found at the upper wall which indicate the existence of the weak zone just after the first baffle. It is also found that when the flow approaches the second baffle, the velocity of flow increases at the upper wall near the second baffle, but it decreases at the lower part of the second baffle. Furthermore, in the cases-[1-3](b), it is found that the flow acceleration is highly influenced at the tip of the second baffle for all of the cases.
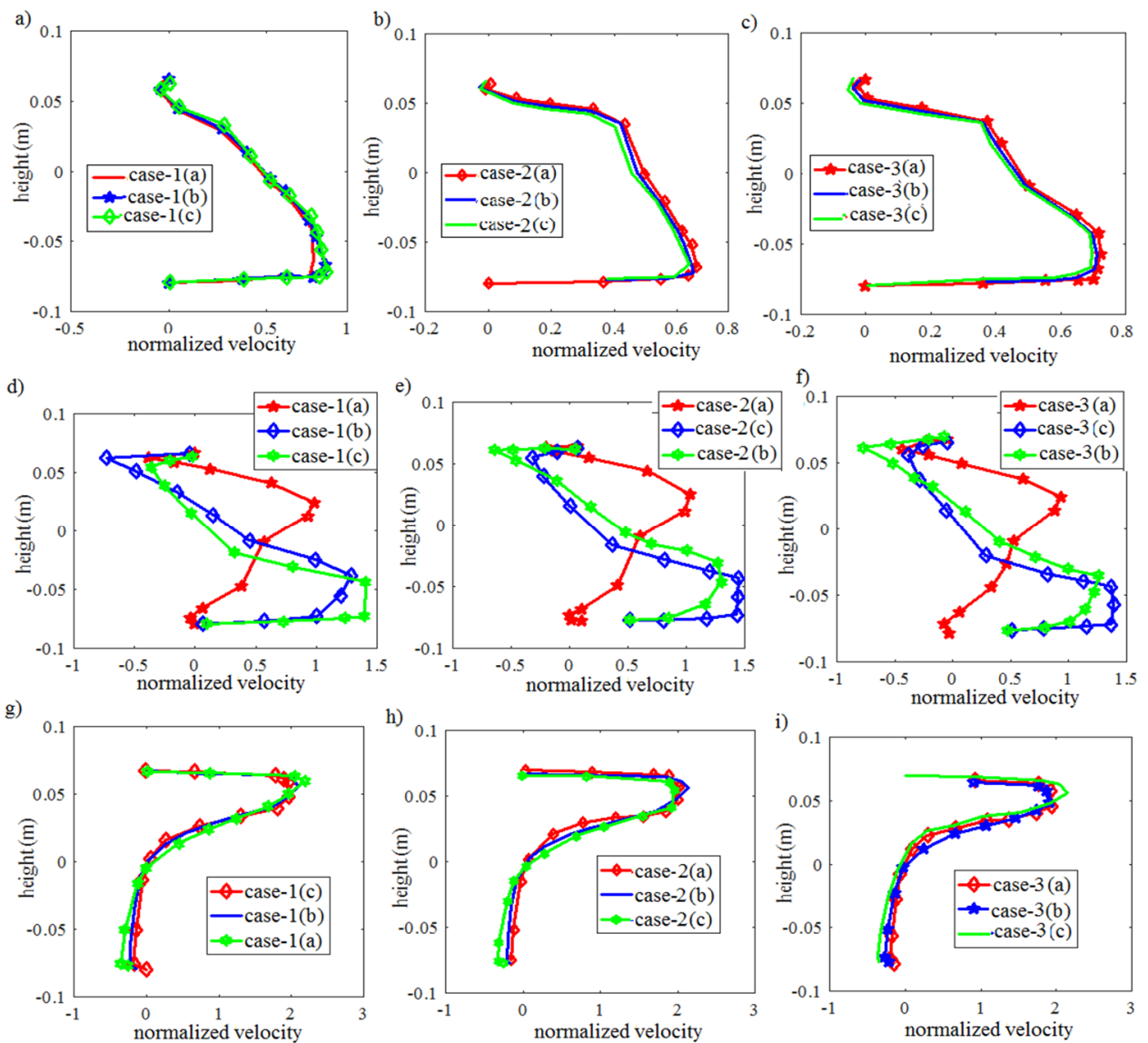

Fig. 3. Velocity profiles at $x=0.189 \mathrm{~m}$ (a-c), $0.289 \mathrm{~m}$ (d-f) and $0.535 \mathrm{~m}$ (g-i) at $R e=5,000$ 
In all the cases, at $x=0.535 \mathrm{~m}$, flow velocity reaches its maximum value, which is more than 4 times of the inflow velocity. As a consequence a large vortex appears near the second baffle which causes the variation in velocity values. From Table 1a and Figures 3a-i, it is found that in the presence of trapezoidal baffles, for baffle distance $l 3$ [case-3(c)], provides maximum velocity as compared to rest, and the minimum velocity value is attained in the case of triangular baffles [case-2].

Table 1(a-b). Analysis of the results in different cases

\begin{tabular}{|c|c|c|c|c|c|c|c|}
\hline \multicolumn{4}{|c|}{ a. maximum velocity $[\mathrm{m} / \mathrm{s}]$ at $R e=5000$} & \multicolumn{3}{c|}{ b. at different li of case-3 at $R e=20,000$} \\
\hline type & case-1 & case-2 & case-3 & $l i$ & $N u_{\text {avg }}$ & $F$ & TEF \\
\hline a & 2.03 & 1.98 & 2.07 & $l 1$ & 475 & 0.0115 & 1.15 \\
\hline b & 2.12 & 2 & 2.19 & $l 2$ & 520 & 0.065 & 1.28 \\
\hline c & 2.20 & 2.01 & 2.25 & $l 3$ & 600 & 0.115 & 1.6 \\
\hline
\end{tabular}

\subsection{Profile of $C_{f}$ and $N u$}

Thermal enhancement leads to less $C_{f}$ which causes the absolute pressure drop to increase. Along the heated upper wall, variations of $C_{f}$ have been studied at $R e=5000$ for the cases 3(a-c). In the intermediate zone between two baffles, the highest friction values are raised due to the existence of a recirculation zone. It is observed that in the upstream ahead of the first baffle, $C_{f}$ becomes low due to the absence of baffle, and the values of $F$ increase in the intermediate zone of the baffles and after the second baffle, these values decrease sharply due to reduction in the contact area of the flow with the hot wall surface. It has been found in Figure $4 \mathrm{a}$ that for the case-3(c), the $C_{f}$ values becomes maximum as compared to rest. In addition, as the primary flow path turns sharply, intermolecular friction between the air and the walls on the other side causes the increase in $F$. The profiles of $F$ have been shown in Figure 4b for different values of $R e$. It is found that the increase in $R e$ causes the decrease in $F$. These decrease rates sharply increase due to the presence of baffles, but the value of $N u_{x}$ increases in between the baffles and near the outlet of the channel. As a consequence, for heat exchange, more time is needed due to the existence of vortex shedding. Figure $4 \mathrm{c}$ presents the profile of $N u_{x}$ for all the cases. Figures $4 \mathrm{c}-\mathrm{d}$ show that the length of recirculation flow attains its maximum value over a smooth region. Due to the change in the flow direction, caused by the baffles, the value of temperature becomes high. Finally, a linear relationship has been found in all the three cases, and it has been concluded that case-3(c) provides the highest $N u_{\text {avg. }}$. With the variation of $R e$, Figure $4 \mathrm{e}$ depicts the variation of $T E F$ for different cases. It has been found that the value of $T E F$ reduces with the increase in the value of $R e$. It has also been found that the TEF increases with the increase in inter baffle spacing [case-3(c)]. Furthermore, at $R e=5000$ and in case-3(c), i.e., at $l 3=0.213 \mathrm{~m}$, it is found that the value of $T E F$ becomes $40 \%$ higher than the case of $l 1=0.071 \mathrm{~m}$. 
The results of the whole study, in terms of $F, N u_{\text {avg }}$ and $T E F$, have been presented in Table 1b.
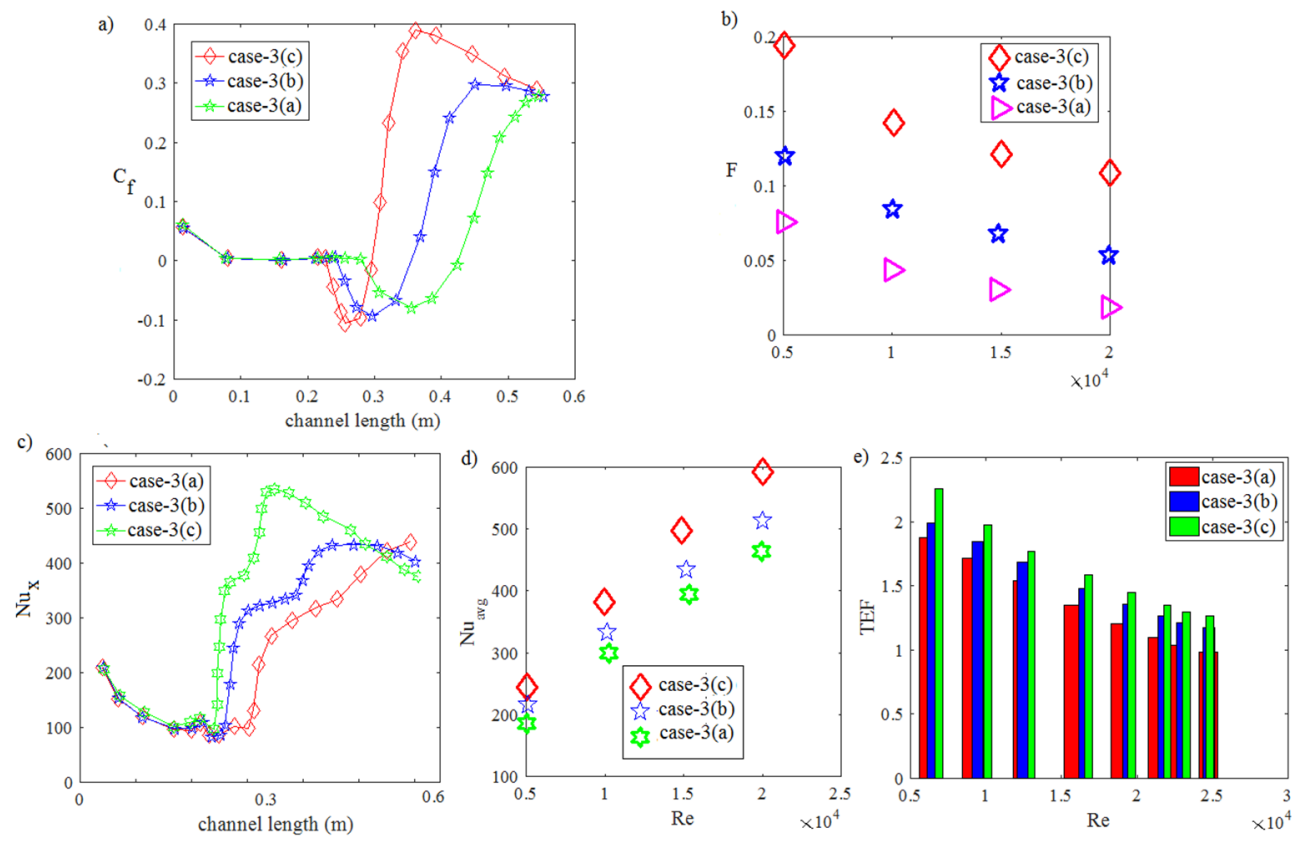

Fig. 4. Profiles of $C_{f}$ (a), $N u_{x}$ (c) at $R e=5000, F$ (b), $N u_{a v g}$ (d) and $T E F$ (e) vs $R e$

\section{Conclusions}

The turbulent air hydro-thermal phenomena have been studied in a rectangular channel with different types of baffle. The problem formulated in this work is a simplification of the baffle geometry used in heat exchangers for shell-and-tube. The effect of inter baffle spacing on flow phenomenona and thermal behavior has also been discussed.

It is revealed that the increase of $R e$ causes the increase of $N u_{\text {avg }}$ and vortex shedding leads to the existence of low and high-pressure regions. In addition, after the second baffle, the rate of heat transfer enhances due to the existence of the vortex region. Furthermore, at $R e=20,000, l 3=0.213 \mathrm{~m}$ and in presence of trapezoidal baffles, it is found that the value of TEF becomes approximately $40 \%$ higher than that of at $l 1=0.071 \mathrm{~m}$. It is also found that the value of $T E F$ increases with the increase in $R e$.

Lastly, an inverse relationship has been found between inter baffle spacing and the heat transfer rate. The above results indicate that the presence of baffles into the flow, and the rate of heat transfer increased highly in a heat exchanger tube. 


\section{Acknowledgments}

Authors wish to thank the reviewer for his valuable suggestions on the improvement of this work and Mr. Sandip Saha thankfully acknowledges the financial support received from MHRD, India.

\section{References}

[1] Yang, Y.T., \& Hwang, C.Z. (2003). Calculation of turbulent flow and heat transfer in a porous baffled channel. International Journal of Heat and Mass Transfer, 46(5), 771-780.

[2] Saffar, A.M., \& Damangir, E. (1995). A general correlation for determining optimum baffle spacing for all types of shell and tube exchangers. International Journal of Heat and Mass Transfer, 38(13), 2501-2506.

[3] Founti, M., \& Whitelaw, J.H. (1985). Shell-side flow in a model disc-and-doughnut heat exchanger. Experiments in Fluids, 3, 293-305.

[4] Pandey, L., \& Singh, S. (2021). Numerical analysis for heat transfer augmentation in a circular tube heat exchanger using a triangular perforated Y-shaped insert. Fluids, 6, DOI: 10.3390/ fluids6070247.

[5] Nagarajan, P.K., \& Sivashanmugam, P. (2011). Heat transfer enhancement studies in a circular tube fitted with right-left helical inserts with spacer, World Academy of Science, Engineering and Technology. Int. J. Mech. Mechatron Eng., 5, 2091-2095.

[6] Gururatana, S., \& Skullong, S. (2019). Experimental investigation of heat transfer in a tube heat exchanger with airfoil-shaped insert. Case Stud. Therm. Engin., 14, 100462.

[7] Tang, X.Y., \& Zhu, D.S. (2012). Experimental and numerical study on heat transfer enhancement of a rectangular channel with discontinuous crossed ribs and grooves. Chin. J. Chem. Eng., 20(2), 220-230.

[8] Sripattanapipat, S., \& Promvonge, P. (2009). Numerical analysis of laminar heat transfer in a channel with diamond-shaped baffles. Int. Commun Heat Mass Transfer, 36(1), 32-38.

[9] Habib, M.A., Mobarak, A.M., Sallak, M.A., Abdel, H., \& Affify, R.I. (1994). Experimental investigation of heat transfer and flow over baffles of different heights. Journal of Heat Transfer, $116(2), 363-368$.

[10] Siddiqui, M.H., \& Kamran, N. (2007). Heat transfer augmentation in a heat exchanger tube using a baffle. International Journal of Heat and Fluid Flow, 28(2), 318-328.

[11] Demartini, L., Crlos, V., Horacio, A., \& Möller, S.V. (2004). Numeric and experimental analysis of the turbulent flow through a channel with baffle plates. Journal of the Brazilian Society of Mechanical Sciences and Engineering, 26(2), 153-159.

[12] Saha, S., Biswas, P., \& Nath, S. (2020). Numerical simulations of Newtonian fluid flow through a suddenly contracted rectangular channel with two different types of baffle plates. Soft Computing, 25, 9873-9885.

[13] Saha, S., Das, N.A., Biswas, P., \& Raut, S. (2021). Numerical simulations of turbulent airflow phenomena and characteristics of heat transfer through a rectangular microchannel with mixed type baffles. International Journal of Fluid Mechanics Research, 48(3), 1-16.

[14] Saha, S., Biswas, P., Raut, S., \& Das, A. (2021). Convective heat transfer of laminar nano-fluids flow through a rectangular micro-channel with different types of baffle-corrugation. Int. J. Computational Methods in Engineering Science and Mechanics, 22(2), 1-13.

[15] Saha, S., \& Das, A. (2021). Flow bifurcation phenomena of shear-thinning and Newtonian fluids in a rectangular channel in presence of intermediate steps: using Carreau-Yasuda model. J. Applied Fluid Mechanics, 14(4), 1283-1293. 
[16] Spalding, D.B. (1974). The numerical computation of turbulent flow. Comp. Methods Appl. Mech. Eng., 3, 269-280.

[17] Dittus, F., \& Boelter, L. (1985). Heat transfer in automobile radiators of the tubular type. Int. Commun Heat Mass Transfer, 12(1), 13-22.

[18] Petukhov, B.S. (1970). Advances in Heat Transfer. Academic Press, 6, 503-504.

[19] Yu, C., Zhang, H., Zeng, M., Wang, R., \& Gao, B. (2020). Numerical study on turbulent heat transfer performance of a new compound parallel flow shell and tube heat exchanger with longitudinal vortex generator. Applied Thermal Engineering, 164, 114-129.

[20] Bicer, N., Engin, T., Yas,ar, H., Büyükkaya, E., \& Aydın, A. (2020). Design optimization of a shell-and tube heat exchanger with novel three-zonal baffle by using CFD and taguchi method. International Journal of Thermal Sciences, 155, 106-117.

[21] Wang, X., Zheng, N., Liu, Z., \& Liu, W. (2018). Numerical analysis and optimization study on shell-side performances of a shell and tube heat exchanger with staggered baffles. International Journal of Heat and Mass Transfer, 124, 247-259.

[22] Saha, S. (2021). Numerical Simulation of Turbulent Flow Through a Sudden expansion Channel: Comparison Between Three models. Lecture Notes in Mechanical Engineering, 222, 49-56.

[23] Saha, S., Biswas, P., Das, N.A. \& Raut, S. (2021). Analysis of heat transfer characteristics through an rectangular enclosure. Mater. Today: Proc., DOI: 10.1016/j.matpr.2021.04.191. 